\title{
Integrated Location Management and Location-Aided Routing System for Mobile Ad-Hoc Networks
}

\author{
Abstract: We propose an integrated location management and location-aided \\ routing system which organizes the Mobile Ad-hoc Network (MANET) into a \\ two-level hierarchy with the help of Voronoi diagrams. The location \\ information of mobile nodes in a Voronoi zone is summarized using Bloom \\ Filters and distributed among the location servers in the zones. The integrated \\ system has the property that the destination's location is learned during routing \\ in the overlay network of the hierarchy. Theoretical analysis and simulation \\ results have shown that the proposed system reduces the routing time by about \\ $30 \%$ over traditional location-aided routing techniques for transaction-type \\ traffic with only small management overhead and low storage requirement.
}

\section{Introduction}

Mobile Ad-hoc Networks (MANET) are becoming increasingly popular because of their ability to self-organize a set of nodes into a network without needing a pre-established network infrastructure [12]. A MANET permits a mobile node to access other nodes that are not within its direct transmission range. This is accomplished using a multi-hop route through intermediate nodes which means that each node in a MANET needs to act as a router as well. Routing in a MANET is inherently complex and requires different approaches from those used in the traditional fixed Internet infrastructure. A major class of routing protocols for MANETs are the location-aided routing protocols [3] [15] [24].

Existing location-aided routing protocols are strongly dependent on the existence of a location management system to enable the storage, distribution, query, and update of the nodes' location information. The routing protocols and the location management systems are generally treated as disjoint subsystems. Typically a location query precedes the actual data transfer. A problem with this approach is that the location query takes time to complete. Not only will this add to the routing delay, the location information obtained may be out-of-date by the time and even during the actual data transfer. Thus an integrated approach that 
combines routing with location query is desirable.

In this paper, we present an integrated location management and routing system that overcomes the afore-mentioned problems. The major characteristics and advantages of the proposed system are:

1. The location query and location-aided packet routing are performed at the same time by piggybacking the data in the location query. This eliminates the query time overhead in traditional systems.

2. The location information of a mobile node is stored in nearby location servers only. This guarantees faster update and lower update overhead. By contrast, in many other systems, the location of a node is stored in a server far away [23] [9], which increases the probability of outdated data and higher update overhead.

3. Our approach yields a path close to the shortest path without incurring significant overhead. Since the database containing location information is distributed among the location servers, storage requirement is small for each individual server. This contrasts with traditional systems using central databases.

The rest of the paper is structured as follows: Section 2 contains a short survey on related work. Section 3 describes the details of the integrated location management and routing system. In Sections 4 and 5, the performance of the system is analyzed theoretically and evaluated by simulations respectively. Finally, Section 6 concludes the paper.

\section{Related work}

Routing algorithms in a MANET can be classified into proactive and reactive protocols. In the proactive approach, routing information containing paths to all possible destinations is stored at each mobile node, which may become outdated due to frequent link failures and topology changes in the MANET [12]. In such cases the reactive approach is preferred. In this approach, a multi-hop path from the sender to the receiver is constructed only when needed. Surveys on these routing algorithms can be found in [19] and [22]. An important group of reactive protocols use location information about the mobile nodes to guide path construction 
[3] [13] [15] [24]. In these algorithms, the destination's location information is used to restrict packet forwarding to within a small region, thus improving efficiency and overhead compared to flooding. The location-aided routing algorithms obtain the destination's location using a location management system which is typically disjoint from the routing mechanism. Designing efficient, scalable location management systems has attracted considerable attention in recent years [6][9][16][23]. In all of these schemes, location query precedes the actual data routing. As mentioned, this will increase the delay time for small data transfers, and it is possible that the location information becomes outdated by the time the actual data transfer is initiated.

This problem has been noted by other researchers. In particular, the EASE system [8] tries to overcome this problem by requiring each mobile node to record the last node it has encountered. The packets are routed toward the destination using the information from these records. However, EASE relies on mobility diffusion to work, which means it will fail to route the packets when node mobility is low, thus hampering its generality.

In wired networks, the landmark routing approach [25] creates a hierarchy of overlays on top of the underlay network. A few nodes are selected recursively to represent a group of nodes at various levels depending on the radius of the area. The predefined hierarchical address of each node reflects its position within the hierarchy and helps find a route to it. Each node knows the routes to all the nodes within its hierarchical partition. Moreover, each node knows the routes to various landmarks at different hierarchical levels. Packet forwarding is consistent with the landmark hierarchy and the path is gradually refined from the top level hierarchy to lower levels as a packet approaches its destination. The idea of hierarchical landmark routing is also used in the context of ad-hoc networks [21]. However, the approach is a direct transplantation from wired networks and no location information is used to guide packet routing.

Two major ideas that underpin our approach is the use of Delaunay triangulation and Bloom filters. Unlike other Delaunay-based schemes [17], our system does not require the Delaunay triangulation to be computed since a mobile node can easily determine the nearest server by the beacon packets sent from the servers. Moreover, our use of Bloom filters is inspired by the 
IP address system in the Internet. The first part of each IP address determines the subnet to which the IP address belongs. When a packet arrives at a router, the router only needs to look up its routing table for the subnet address of the destination. Therefore, all packets destined to a subnet can go along the same path directed by the same subnet address. Although this hierarchical routing is efficient for the Internet, it cannot be directly transplanted for use in the ad-hoc network environment. The major problem is that in an ad-hoc network, the nodes within the same virtual zone do not have the same IP address prefix as in the Internet. This problem is solved in our proposed system using Bloom Filters (see Section 3).

\section{The integrated location management and routing system}

Our integrated location management and routing approach is designed to:

- Exploit the proximity between the node and the nearby location servers to store its location information so as to enable piggybacking the location query to data routing.

- Not rely on central storage or administration so it is robust against frequent node or link failure which is characteristic of MANET.

The above two points are achieved by a partition scheme based on Delaunay triangulation.

- Minimize overhead of information exchange among the location servers by keeping the information concise.

This is achieved by a messaging scheme based on Bloom Filters.

The details of our integrated system are explained below. 


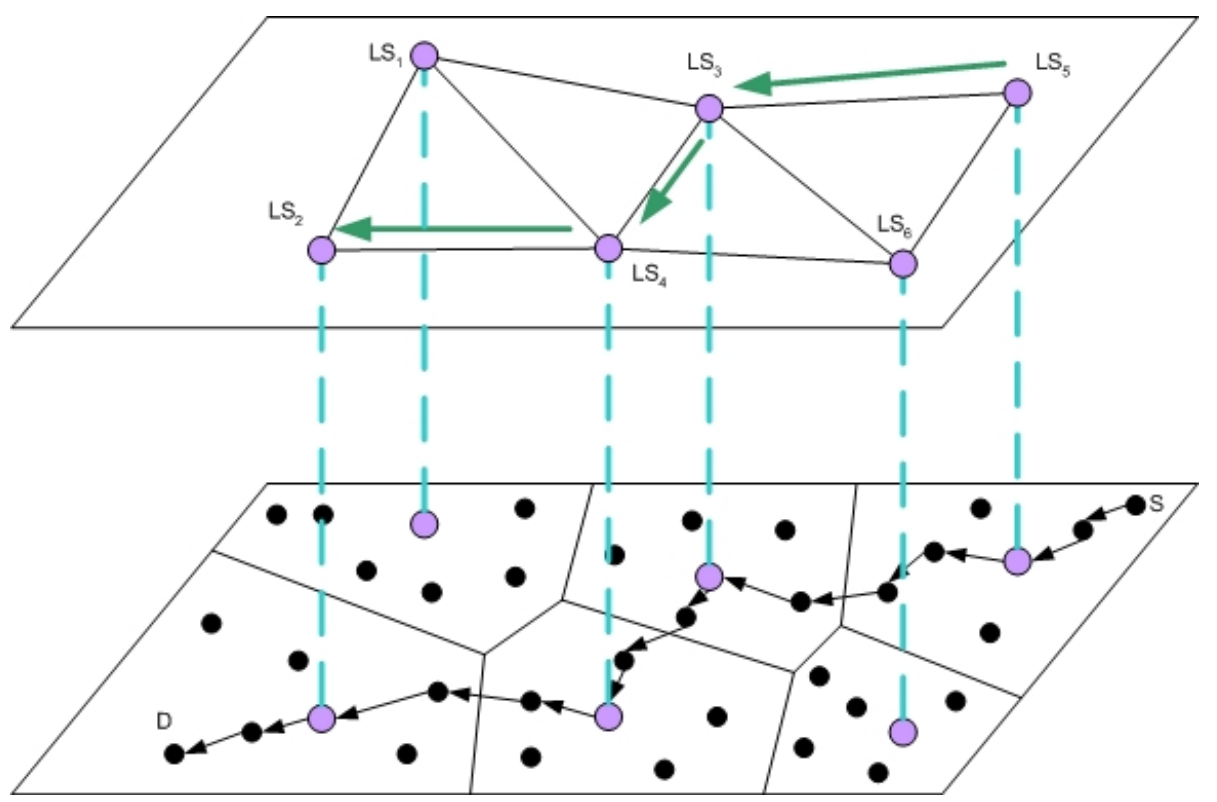

Fig. 1. Overview of the integrated location management and routing system.

\subsection{Delaunay triangulation overlay network}

In our integrated system, a subset of the nodes in the MANET is designated as location servers ( $L S_{1}$ to $L S_{6}$ in Fig. 1, which are represented by large dots). The upper layer of Fig. 1 is an overlay network formed by establishing virtual links among the location servers. The links in the overlay network correspond to paths in the underlay ad-hoc network, which are established and maintained using a location-aided protocol. The functions of the overlay network are as follows: (1) The location servers communicate with each other to exchange their own location information and other control messages. (2) The overlay network is also used during routing to establish paths among the location servers. The problem of how to select the location servers is out of the scope of this paper. We only require that the location servers be roughly uniformly distributed in the ad-hoc network. Several protocols, such as the Landmark Election protocol in the Multiple Landmark Routing Scheme [10] and the GDMS group leader selection algorithm [18], can be used to select the location servers.

The use of location servers is motivated by the two major types of ad hoc networks: low mobility ad hoc network, and high mobility ad hoc network. Campus ad hoc network is an example of the low mobility ad hoc network, composed of quasi-static nodes which move 
within an office room or around a certain gathering point (e.g. a coffee shop). It is obvious that EASE [8] does not work well in such a low mobility network because it is based on the list of last encountered nodes. Having a location server for an office room or a gathering point is reasonable and convenient for packet routing for the low mobility environment. Battlefield ad hoc network represents a typical high mobility ad hoc network, with soldiers and tanks moving roughly toward the same direction. Vehicular ad hoc network is another example of the high mobility ad hoc network, with vehicles moving at high speed. In these high mobility ad hoc networks, having location servers is advantageous since the relative locations between moving nodes and the servers are roughly maintained. Again, EASE does not work well in this environment.

A location server has links only with nearby location servers that are connected to it in the Delaunay triangulation [5] of the location servers. The Delaunay triangulation of a set of nodes $A$ is defined as a triangulation such that for each circumscribing circle of a triangle formed by three vertices in $A$, no vertex of $A$ is in the interior of the circle. The Delaunay triangulation is the dual of the Voronoi diagram in that for each pair of nodes whose Voronoi cells are neighbors, there is an edge connecting them in the Delaunay triangulation.

Using the Delaunay triangulation has the following advantages: First, the average degree of a location server in the overlay network is small. Since the number of edges of the Delaunay triangulation is bounded by $3 m-3$, where $m$ is the number of location servers, the average degree of the location servers is bounded by 6 . Second, it is guaranteed that virtual links exist only between location servers that are in close proximity to each other. This ensures that the corresponding paths in the underlay ad-hoc network are short in terms of the number of hops, which makes it easier to maintain the paths.

\subsection{Voronoi cell virtual zone}

A virtual zone is assigned to each location server, which corresponds to the Voronoi cell [1] of the location server (See Fig. 2). Therefore, each location server contains the location information of all the nodes within its Voronoi cell. By the definition of the Voronoi diagram [1], it is guaranteed that the location information of a node is sent to the nearest location 
server. A mobile node periodically updates its location information to the $k$ nearest location servers, where $k$ is a system parameter. When $k=1$, a location server is storing location information of all the mobile nodes within its Voronoi cell. A larger value of $k$ can be used to alleviate the data availability problem since the location information is still available at other location servers even if a location server fails. In Sections 4 and 5, we show by theoretical analysis and simulation experiments respectively that $k=2$ or 3 is appropriate considering the tradeoff between storage and data availability. In the subsequent subsections, we describe our system assuming $k=1$ for simplicity.

When a mobile node moves across a Voronoi cell border, it sends a JOIN message followed by the location information to the new location server. At the same time, a LEAVE message is sent to the previous location server so that the corresponding outdated location information can be deleted from the server's database.

\subsection{Information messaging using Bloom Filters}

Bloom Filters are an efficient, lossy way of describing sets. A Bloom Filter is a bit-vector of length $w$ with a family of hash functions, each of which maps from elements of the associated set to an integer in $[0, w)$. To form the description of a set, each element in the set is hashed by the family of hash functions, and the bits in the vector corresponding to the hashed results are set. To determine whether the set represented by a Bloom Filter contains a specific element, that element is hashed by the hash functions and the corresponding bits in the filter are examined. If any of the bits is not set, the represented set definitely does not contain that element (see Fig. 2 for an example). If all the bits checked are set, the set may contain the element. There is still a non-zero probability that it does not. This case is called false positive. The rate of false positive of a Bloom Filter is a well-studied linear function of its width, the number of hash functions and the cardinality of the represented set. We will discuss the problem of minimizing false positive rate in Section 4. 


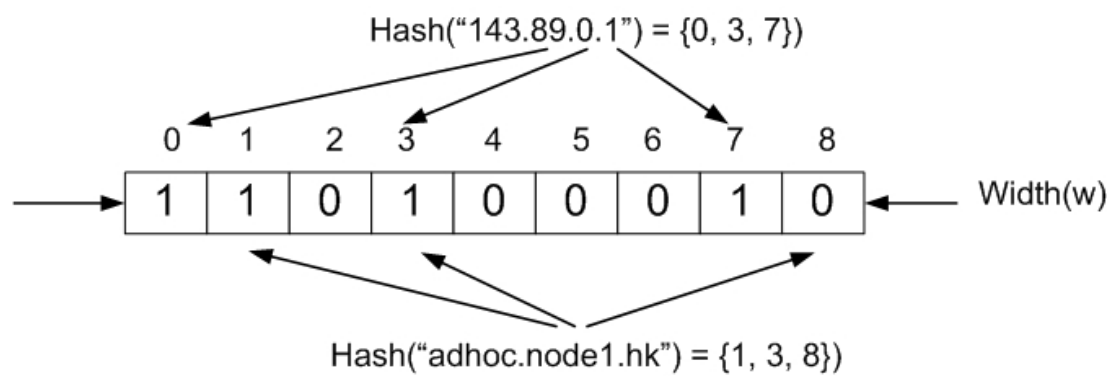

Fig. 2. A Bloom Filter example. Three hash functions are used. When the mobile node with the name "ad-hoc.node1.hk" is checked, we know definitely that it is not served by the location server since bit 8 is false.

In our integrated scheme, each location server uses a Bloom Filter to represent the set of mobile nodes it serves. Each server keeps the location information of roughly $N^{1 / 2}$ mobile nodes, where $N$ is the number of nodes in the network. To facilitate queries, the location information contained by a server needs to be made available to the others. One intuitive approach would be flooding the Bloom Filter to the other servers. Since each server contains the Bloom Filters of all servers eventually, when a packet arrives, the server can easily determine which server has the information for the destination. The packet can then be routed in the overlay network to that location server. However, this approach requires each location server to keep $m$ Bloom Filters, where $m$ is the number of location servers in the ad-hoc network.

Our scheme requires each location server to maintain only $d$ Bloom Filters, where $d$ is the degree of the server in the overlay network. Since the average degree of the location server is bounded by 6 , this greatly reduces the storage requirement.

Each location server associates a Bloom Filter with each outgoing link in the overlay network. When a location server $C$ needs to distribute its Bloom Filter, it sends the Bloom Filter along the shortest path tree in the overlay network. Each time the Bloom Filter arrives at another location server $A$ via a specific link $(B, A)$, the associated Bloom Filter of the outgoing link $(A$, $B$ ) of location server $A$ is aggregated by the bit-wise OR operation of the two.

Fig. 3 shows an example of Bloom Filter distribution. Location server $L S_{2}$ contains location 
information of mobile node "143.89.0.1" and its Bloom Filter sets the corresponding bits $\{0$, $3,7\}$ to 1 . When the Bloom Filter is distributed along the shortest path tree as denoted by the arrows, the Bloom Filters of the following links are updated: $\left(L S_{4}, L S_{2}\right),\left(L S_{1}, L S_{2}\right),\left(L S_{3}, L S_{4}\right)$, $\left(L S_{5}, L S_{3}\right)$, and $\left(L S_{6}, L S_{4}\right)$. Note that after location server $L S_{1}$ distributes its Bloom Filter along the dotted arrows, the Bloom Filter associated with link $\left(L S_{6}, L S_{4}\right)$ is the aggregation of the Bloom Filters of both $L S_{1}$ and $L S_{2}$.

When new mobile nodes enter the Voronoi cell of a location server, the corresponding Bloom Filter needs to be updated by turning on the hashed bits. The server only needs to calculate the changed bits of the Bloom Filter and sends them to the other location servers. This is known as differentiated update. It is important to note that when a mobile node leaves a Voronoi cell, the corresponding bits cannot be simply reset because other nodes may be hashed to those bits. To resolve this problem, either the Bloom Filter needs to be rebuilt periodically, or a counter should be associated with each bit of the Bloom Filter. The latter approach is called a counting Bloom Filter.

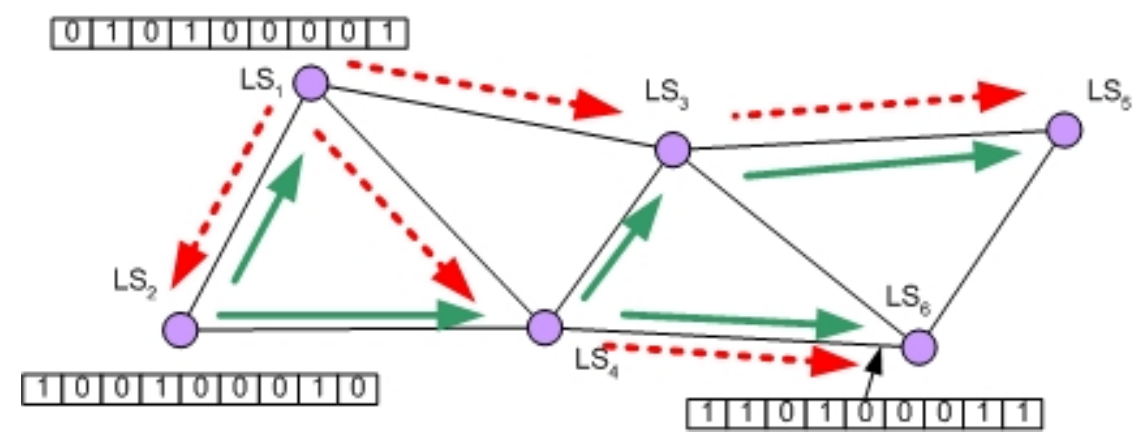

Fig. 3. Bloom Filter distribution. We assume only location server $L S_{1}$ and $L S_{2}$ are distributing their Bloom Filters.

\subsection{Location-aided routing}

The routing of a packet from a sender $S$ to a destination $D$ is divided into three phases. First, the sender sends the packet to the nearest location server. The location server, with the help of Bloom Filters, determines where to route the packet in the overlay network formed by the location servers. Hop by hop, the packet is forwarded to the location server closest to the destination via the overlay network. Finally, the location server forwards the packet to the destination. A local routing protocol (LRP) is used in the first phase (from the sender to its 
location server) and the third phase (from the destination's location server to the destination). Another Inter-Location-Server Protocol (ILSP) is used for routing among the location servers in the overlay network guided by information sharing using Bloom Filters. As an example, in Fig. 1, when the source node $S$ needs to send a message to the destination node $D$, the message is first sent to the nearest location server $L S_{5}$ using the LRP protocol. The message is then routed in the overlay network to location server $L S_{2}$ which contains the location information of the destination node $D$. The LRP protocol is employed again to route the message from $L S_{2}$ to $D$. The virtual path in the overlay network and the corresponding path in the underlay network are shown by arrows in Fig. 1.

Since a location server broadcasts its location information to all the mobile nodes in its Voronoi cell, the LRP protocol can use the location information to guide packet routing. Therefore, any location-aided routing protocol can be employed. We use the LAR protocol proposed in [15] in our prototype implementation.

For the ILSP protocol, the objective is to route the packet to the location server containing the destination's location information. When a location server receives a packet from a mobile node in its Voronoi cell with destination address desti_name, it first checks its own Bloom Filter to see if it has the destination's location information there ${ }^{1}$. If so, the location database is consulted and the packet is passed to the destination using the LRP protocol. Otherwise, the location server examines the Bloom Filters of all its outgoing links. If one of the filters matches, the packet is forwarded to the neighbor along the matched link. If multiple links match, the first one is chosen. The packet is relayed in this way until it reaches the target location server.

Take Fig. 3 as an example. Suppose a mobile node in $L S_{6}$ 's Voronoi cell needs to send a packet to another node "ad-hoc.node1.hk”. Here we suppose that "ad-hoc.node1.hk” is served by location server $L S_{1}$ and it hashes to $\{1,3,8\}$. The source node first sends the packet to its nearest location server $L S_{6}$ using the LRP protocol. $L S_{6}$ then checks its own Bloom Filter and

\footnotetext{
${ }^{1}$ An alternative way is to check the exact list of the nodes in the virtual zone. However, this increases the overhead of checking.
} 
finds that it does not have information about the destination node. It then checks the Bloom Filters of its three outgoing links. Link $\left(L S_{6}, L S_{4}\right)$ is selected since all the three bits are set. The packet is forwarded to $L S_{4}$ and its outgoing link $\left(L S_{4}, L S_{1}\right)$ is chosen. When the packet reaches $L S_{1}$, ILSP finds that it is the target location server and the packet is routed to the destination node "ad-hoc.node1.hk" using the LRP protocol.

Because of false positive, it is possible that the packet may reach a location server whose Bloom Filter claims to contain the destination while the actual location database does not have it. When this happens, one can simply give up or forward the packet to one of its neighbors. To avoid infinite forwarding along loops, the number of traversed hops in the overlay network is recorded so that the packet can be discarded when the number exceeds a specified threshold. An alternative approach is to attach a list of all servers visited so far with each packet. This list is used to prevent the packet from being forwarded to a server it has already visited.

\section{Theoretical analysis}

In this section we analyze several interesting metrics for the proposed scheme.

\subsection{Optimal number of location servers}

Let $N$ denote the number of nodes in the ad-hoc network and $m$ be the number of location servers. We set $m=\Theta\left(N^{1 / 2}\right)$ to optimize system performance. This is justified as follows. By assuming the communication sessions are between pairs of nodes arbitrarily situated throughout the network, it has been shown in [14] that the average throughput per node is $\Theta\left(N^{1 / 2}\right)$. Assume that the network is uniformly partitioned into Voronoi cells, we can argue that the average number of nodes in each virtual zone is $\mathrm{N} / \mathrm{m}$. Then the average throughput per node of a local virtual zone is $R_{\text {local }}=\Theta\left([N / m]^{-1 / 2}\right)$. The per-node throughput in the overlay network is given by $R_{\text {overlay }}=\Theta\left(\mathrm{m}^{-1 / 2}\right)$. Since traffic across virtual zones are switched at the location servers from local zone to the overlay networks or vice versa, the portion of $R_{\text {local }}$ sent to other virtual zones via the overlay network should be $(m-1) / m$ assuming uniform traffic distribution. To avoid congestion, this portion should not exceed $R_{\text {overlay }}$. Since we want to achieve the maximum throughput without causing congestion, we have $(m-1) / m R_{\text {local }}=$ 
$R_{\text {overlay, }}$ which gives $m=\Theta\left(N^{1 / 2}\right)$.

In our experiment, we set the number of location servers to be $\left\lfloor N^{1 / 2}\right\rfloor$. In the example of Fig.

1, we have 39 nodes and 6 location servers.

\subsection{Location server availability}

The location information of a mobile node is stored in $k$ location servers. Assume that $p$ is the probability that an individual location server works correctly. Since the data is accessible when at least one location server is available, the data availability is given by the following binomial distribution:

$$
A_{\text {data }}(p)=1-(1-p)^{k}
$$

We define the desired availability, $a$, as the degree of data availability that must be guaranteed in order for the system to be reliable. The following table gives the number of replica location servers, $k$, needed to meet availability requirements for different failure rates. From the table, we can see that if we only choose reliable (i.e., $p>0.75$, which is a reasonable requirement) nodes to act as location servers, only a small number of replicas (2 to 4) are needed to achieve a system with high data availability $(a>0.99)$.

\begin{tabular}{|c|c|c|c|c|c|c|}
\hline Maximum required number of replicas to maintain availability \\
\hline \multirow{2}{*}{$\begin{array}{c}a, \text { (desired } \\
\text { availability) }\end{array}$} & \multicolumn{5}{|c|}{$p$, (individual location server availability) } \\
\cline { 2 - 7 } & 0.5 & 0.75 & 0.9 & 0.95 & 0.99 & 0.999999 \\
\hline 0.5 & 1 & 1 & 1 & 1 & 1 & 1 \\
\hline 0.75 & 2 & 1 & 1 & 1 & 1 & 1 \\
\hline 0.9 & 4 & 2 & 1 & 1 & 1 & 1 \\
\hline 0.95 & 5 & 3 & 2 & 1 & 1 & 1 \\
\hline 0.99 & 7 & 4 & 2 & 2 & 1 & 1 \\
\hline 0.999999 & 20 & 10 & 6 & 5 & 3 & 1 \\
\hline
\end{tabular}

Table I. Data availability with respect to individual location server availability.

\subsection{Path quality}

We now analyze the quality of the path between any two randomly chosen mobile nodes in the ad-hoc network. To facilitate analysis, we assume that all the mobile nodes are uniformly distributed in the area. We further assume that the network is connected and no isolated nodes 
exist in the network.

Assume S and D are two nodes in the ad-hoc network and the path connecting them passes through location servers $L S_{0}, L S_{1}, \ldots, L S_{p}$ in the overlay network. We use $|S, D|$ to denote the Euclidean distance between them.

Lemma 1: The average number of hops, $p$, between any two location servers in the overlay network is $\Theta\left(m^{1 / 2}\right)=\Theta\left(N^{1 / 4}\right)$.

Lemma 2: Define the Delaunay distance between any two location servers as the length of the shortest path in the Delaunay graph connecting the servers. Then the ratio between the Delaunay distance and Euclidean distance of any pair of location servers is bounded by a constant $c$. This constant $c$ is $\pi / 2$ for arbitrary graphs and is reduced to $4 / \pi$ if the locations in the Delaunay graph are generated with respect to the Poisson process [2].

Lemma 3: Denote the length of the path connecting $L S_{0}$ and $L S_{p}$ as $\operatorname{Dist}\left(L S_{0}, L S_{p}\right)$, then

$$
\operatorname{Dist}\left(L S_{0}, L S_{p}\right) \leq c^{2}\left|L S_{0}, L S_{p}\right| \text {. }
$$

Proof:

$\operatorname{Dist}\left(L S_{0}, L S_{p}\right)$ is made up of the path lengths connecting two servers. Remember the path between any two servers is a shortest path, which is shorter than the Delaunay distance if the mobile nodes between them are Delaunay triangulated ${ }^{2}$. Therefore,

$$
\begin{aligned}
\operatorname{Dist}\left(L S_{0}, L S_{p}\right) & \left.=\sum_{\mathrm{i}=0 \text { to } p-1} \text { (length of shortest paths between } L S_{i} \text { and } L S_{i+1}\right) \\
& \leq \sum_{\mathrm{i}=0 \text { to } p-1} \text { (Delaunay distance between } L S_{i} \text { and } L S_{i+1} \\
& \quad \text { if the mobile nodes are Delaunay triangulated) } \\
& \leq \sum_{\mathrm{i}=0 \text { to } p-1} C\left|L S_{i}, L S_{i+1}\right| \\
& \leq c^{2}\left|L S_{0}, L S_{p}\right|
\end{aligned}
$$

Theorem 1: In the average case, given two random nodes $S$ and $D$, the ratio between the path

\footnotetext{
${ }^{2}$ Here we assume that there exists a link between any two mobile nodes in the underlay ad-hoc network if they are connected in the Delaunay triangulation.
} 
adopted by the proposed system, and the Euclidean distance is bounded, i.e.,

$$
\operatorname{Dist}(S, D) \leq c^{2}|S, D|
$$

Proof:

Assume the source node $S$ and destination $D$ are also location servers. The proof then follows the same argument as that for Lemma 3.

In the paper, we are interested in the number of hops used in the path denoted by $v(p a t h(S$, $D))$. It can be seen that $\mathbf{E}[\operatorname{path}(S, D)]=\mathbf{E}[v(\operatorname{path}(S, D))] l$, where $l$ is the average length of the edges on the path. This is also true for the shortest path, i.e., $\mathbf{E}[\operatorname{shortest} \operatorname{path}(S, D)]=$ $\mathbf{E}[v($ shortest path $(S, D))] l$ ', where l' is the average length of the edges on the shortest path. Therefore we have

$$
\begin{aligned}
\mathbf{E}[v(\operatorname{path}(S, D))] & =\mathbf{E}[\operatorname{path}(S, D)] / l \leq c^{2}|S, D| / l \leq \mathbf{E}[\operatorname{shortest} \text { path }(S, D)] c^{2} / l \\
& \leq \mathbf{E}[v(\operatorname{shortest} \text { path }(S, D))] l^{\prime} c^{2} / l
\end{aligned}
$$

Theorem 2: On average, the ratio between the path in our system and the shortest path, with respect to the number of hops, is bounded by a constant $d=c^{2} l ' / l$, where $l$ and $l$ ' are the average Euclidean length of the path in our system and the shortest path respectively.

Theorem 1 shows that the ratio between the path chosen by our system and the Euclidean distance is bounded. In Theorem 2 the ratio with respect to path hops is also bounded. Since in the adhoc network, path delay is directly determined by the number of hops in the path, our path approximates the shortest path very well with respect to path delay.

\subsection{False positive}

The probability of a Bloom Filter false positive error is given by

$$
f=\left(1-(1-1 / b)^{N k}\right)^{k}
$$

where $N$ is the number of mobile nodes in the ad-hoc network, $b$ is the number of bits of the Bloom Filter, and $k$ is the number of hash functions. For large values of $m$, the above formula reduces to 


$$
f \approx\left(1-e^{-N k / b}\right)^{\mathrm{k}} .
$$

First let's suppose $N$ and b are fixed, and we would like to find the optimal number of hash functions, $k$, to minimize the false positive ratio. By solving $\frac{d f}{d k}=0$, we have

$$
k=(b / N) \ln 2
$$

and the corresponding false positive probability is

$$
f=0.5^{k}
$$

Since the Bloom Filters need to be transferred among the location servers in our integrated system, we also want to minimize the size of the Bloom Filter, i.e., the number of bits $b$, to reduce the transmission message size.

\subsection{Storage}

Given a mobile node, the location information of its neighboring nodes is needed to perform location-aided routing. Note that the average degree in a planar graph is bounded by 6. Also, the node must maintain its own location information as well as that of the nearest location server. Therefore, the storage requirement is bounded by $S=8 q$, where $q$ is the size of a location information item.

A location server needs to store three things: (1) Location information of all mobile nodes in its Voronoi cell. On average, $N^{1 / 2}$ items are kept in the location database. (2) A summary of the location information represented by a Bloom Filter. (3) The Bloom Filters associated with each of its outgoing link. These Bloom Filters have the same number of bits as the location server's own summary. The average number of Bloom Filters associated with the links is bounded by 6 .

Therefore, the storage requirement of a location server is bounded by $S=q N^{1 / 2}+7 b$. While in traditional location management systems, the storage requirement is basically proportional to $N$ since centralized location databases are employed.

\subsection{Routing Overhead}

\section{Node registration}


Assume that $f_{R}$ is defined as the frequency per node at which location server registration events occur. Since a location server registration event occurs when a mobile node migrates from one Voronoi zone to another, $f_{R}$ can be expressed simply as a function of the average node speed $\mu$ and the average Voronoi cell area $A_{V}$. Since $A_{V}=\Theta(m)=\Theta\left(N^{1 / 2}\right)$, where $m$ is the number of Voronoi cells in the network, we have:

$$
f_{R}=\Theta\left(\mu / A_{V}^{1 / 2}\right)=\Theta\left(N^{-1 / 2}\right)
$$

Denote the average number of mobile nodes in a Voronoi cell as $N_{V}=\Theta\left(N^{1 / 2}\right)$. To register, a mobile node needs to send a registration packet to the nearest location server. The average number of hops the packet traverses is $h=\Theta\left(N_{V}{ }^{1 / 2}\right)=\Theta\left(N^{1 / 4}\right)$. Since $f_{R}$ applies for all $N$ nodes, the overhead of node registration in the system is given by

$$
\Psi_{\text {REGISTRATION }}=N \cdot f_{R} \cdot h=\Theta\left(N^{3 / 4}\right) .
$$

\section{Node location update}

We assume the frequency that a mobile node updates its location information is $f_{U}$. The update packet needs to traverse $h=\Theta\left(N_{V}{ }^{1 / 2}\right)=\Theta\left(N^{1 / 4}\right)$ hops to reach the location server. Since all $N$ nodes need to update their location information, the node location update overhead is:

$$
\Psi_{\text {UPDATE }}=N \cdot f_{U} \cdot h=f_{U} \Theta\left(N^{5 / 4}\right) .
$$

\section{Bloom Filter update among location servers}

When the Bloom Filter of a location server changes, it needs to be distributed to the other location servers. Since the size of the Bloom Filters is $b$ bits, we assume the message size is $\Theta(1)$. Also note that a Bloom Filter update can only be caused by the node registration event, hence the frequency of Bloom Filter update per location server $f_{B}=N_{v} \cdot f_{R}=\Theta\left(N^{1 / 2} N^{-1 / 2}\right)=$ $\Theta(1)$.

A Bloom Filter update message needs to be distributed to the other location servers using a shortest path tree in the overlay network. The number of edges in the overlay network $h_{\text {overlay }}$ $=\Theta(m)=\Theta\left(N^{1 / 2}\right)$. Note that each of these edges is a path with $h_{\text {underlay }}=\Theta\left(N^{1 / 4}\right)$ mobile nodes 
in the underlay network. Therefore, the overhead of Bloom Filter update is:

$$
\Psi_{\text {BLOOM }}=m \cdot f_{B} \cdot h_{\text {overlay }} \cdot h_{\text {underlay }}=\Theta\left(N^{5 / 4}\right)
$$

\section{Experimental results}

The proposed system was evaluated using the Glomosim simulator [7] . As in the work of Pei et. al. [21], the radio transmission range of each node in the ad-hoc network was 250 meters and the channel capacity was 2Mbits/sec. We also assumed that the network is uniformly distributed in a square area. Two sets of network configurations were used in the simulation. The first set had a fixed network size of $1000 \times 1000$ and the number of nodes was varied from 100 to 1000 in steps of 100 . To study the effect of different network sizes on performance, a second set of network configurations with the same node density but different network sizes (see Table II) was used. Note that node density is defined as the number of nodes per $\mathrm{km}^{2}$.

\begin{tabular}{||l|c|c||}
\hline \hline network size & number of nodes & node density \\
\hline $1000 \times 1000\left(\mathrm{~m}^{2}\right)$ & 100 & 100 \\
\hline $2000 \times 2000\left(\mathrm{~m}^{2}\right)$ & 400 & 100 \\
\hline $3000 \times 3000\left(\mathrm{~m}^{2}\right)$ & 900 & 100 \\
\hline
\end{tabular}

Table II. Network configurations with fixed node density.

\subsection{Mobility model}

The random waypoint model was used to simulate node mobility. In this model, a node randomly selects a destination and moves toward it at a fixed speed. Once the destination is reached, a new destination is selected after a certain pause time. The velocity is uniformly selected from the range $[a-b, a+b]$, where $a$ and $b$ are system parameters that control the average node speed and the speed variation among nodes. In the experiments, $a$ and $b$ were set at $10 \mathrm{~m} / \mathrm{s}$ and $5 \mathrm{~m} / \mathrm{s}$ respectively. Larger values can also be set to reflect a vehicular network. 


\subsection{Performance metrics}

The following metrics were used to evaluate the performance of the integrated system:

(i) path ratio - the ratio between the length of the path adopted by the integrated system and the shortest path.

(ii) routing time ratio - the decrease in routing time compared to traditional location-aided routing where data packets are sent only after the location of the destination is obtained from the location management system. The routing time is the time interval between the time a packet is issued at the source and the time it reaches the destination.

(iii) transmission success ratio - the probability that the packets are transmitted to the destination correctly. Unsuccessful transmission may be caused by temporary unavailability of some location servers or false positive errors of the Bloom Filters.

The first two metrics reflect the efficiency of the integrated system. The last metric is used to evaluate the reliability of the system.

\subsection{System used for comparison}

There are no integrated systems reported in the literature that we are aware of. Therefore, we created a system for comparison with the proposed scheme by combining the UQS location management system [9] with the location-aided routing (LAR) algorithm [15]. They were selected because they are representatives of existing systems and algorithms. We denote this hybrid system as "UQS+LAR" in the rest of the paper.

\subsection{Simulation results}

Unless specified otherwise, the simulation results presented below were obtained by taking the average of 10 runs of simulation experiments.

\subsubsection{Correctness of Bloom Filters}

It is important to note that Bloom Filters serve two purposes in our proposed integrated system. Besides being used by each location server to generate location summaries for all the 
mobile nodes within its zone, it is also associated with each outgoing link of the location server and contains location summaries seen by that link. To evaluate the effect of false positive errors, we need to investigate these two different scenarios separately.

For the first situation, we define a metric called the "server filter correctness ratio" as follows. A node and location server pair $(X, Y)$ are randomly generated. We then check if hashing $X$ 's ID can cause a false positive in Y's Bloom Filter. The operation is performed for each node/server pair in the ad-hoc network and the correctness ratio is calculated.

For the second situation, we define a metric called the "link filter correctness ratio". For each randomly generated node and link pair $(X, Y)$, we check if hashing $X$ 's ID causes a false positive in link Y's Bloom Filter and then calculate the average correctness ratio.

Note that different $b / N$ (i.e., the number of bits in the Bloom Filter over the number of nodes in the ad-hoc network) ratios can affect the correctness ratio. We used six different $b / N$ ratios1, 2, 4, 8, 16 and 32 in our experiments. Also we experimented with four different numbers of hash functions $k-2,3,4$, and 5 . The value of $b / N$ affects the storage space needed for each location server, and the value of $k$ has influence on the computation overhead in node query.

We choose the largest network with 900 nodes randomly distributed in an area of $3000 \times 3000$ $\mathrm{m}^{2}$ square in this simulation. The IP addresses of the mobile nodes were randomly chosen from the entire 32-bit IPv4 address space.

The results of this set of simulation are summarized below.

\begin{tabular}{l|l|l|l|l} 
& $k=2$ & $k=3$ & $k=4$ & $k=5$ \\
\hline$b / N=1$ & 0.7964 & 0.8113 & 0.8035 & 0.7720 \\
\hline$b / N=2$ & 0.8873 & 0.9235 & 0.9288 & 0.9276 \\
\hline$b / N=4$ & 0.9162 & 0.9506 & 0.9520 & 0.9536 \\
\hline$b / N=8$ & 0.9502 & 0.9542 & 0.9551 & 0.9545 \\
\hline$b / N=16$ & 0.9509 & 0.9560 & 0.9573 & 0.9571 \\
\hline$b / N=32$ & 0.9511 & 0.9571 & 0.9577 & 0.9575
\end{tabular}

Table III. Server filter correctness ratio.

\begin{tabular}{l|l|l|l|l} 
& $k=2$ & $k=3$ & $k=4$ & $k=5$ \\
\hline$b / N=1$ & 0.7757 & 0.7988 & 0.7897 & 0.7720 \\
\hline$b / N=2$ & 0.8685 & 0.9102 & 0.9138 & 0.9121 \\
\hline$b / N=4$ & 0.8960 & 0.9413 & 0.9420 & 0.9433 \\
\hline$b / N=8$ & 0.9413 & 0.9454 & 0.9459 & 0.9456 \\
\hline$b / N=16$ & 0.9415 & 0.9458 & 0.9463 & 0.9460 \\
\hline$b / N=32$ & 0.9424 & 0.9464 & 0.9572 & 0.9569
\end{tabular}

Table IV. Link filter correctness ratio 
It is seen that the link filter correctness ratio is slightly lower than the server filter correctness ratio. This is because link Bloom Filters are generally the sum of several Bloom Filters which increases the chance of collision among bits.

Among different $b / N$ settings, the ratio of 1 had the worst performance, while 2 was only slightly worse than 4 and 8 . As the ratio increased to 16 and 32, no significant improvement in performance was observed. This suggests that further increasing the number of bits of the Bloom Filters will not improve performance significantly. Allocating only 4 bits on average for each node in the network already yields acceptable performance.

The simulation results of different $k$ settings did not show much variation when $k$ went beyond 3 .

It is important to note that using a large number of hash functions does not necessarily guarantee higher correctness ratio since they may cause more bit collisions when the Bloom Filters are saturated. From Table III and Table IV, we can see that for each $b / N$ ratio there exists an optimal value of $k$ which achieves the maximum correctness ratio. This justifies the analyses in Section 4.4.

The simulation results in this subsection show that with the use of Bloom Filters, the proposed integrated scheme is able to represent the set of nodes in the adhoc network without much storage requirement. This is in contrast with the large ID name space needed in other approaches. Moreover, system parameters can be configured to strike a balance between correctness ratio and storage requirement.

\subsubsection{Quality of paths}

The metric of path ratio was used to evaluate the quality of the actual paths generated by our integrated system.

The network spanning area was fixed at $1000 \times 1000 \mathrm{~m}^{2}$, and the number of nodes in the network was varied from 100 to 1000 . The length of the path, defined as the sum of Euclidean distances of the links along the path, was compared to the length of the shortest path from the source to the destination found by Dijkstra's algorithm.

The simulation results are shown in Fig. 4. It is seen that the ratio varies in the range of 
1.2 1.4, which means that the paths adopted by our system was only 20 to $40 \%$ longer than the shortest paths. Note that the ratio was higher when network sizes were small. This is because the paths have to traverse in the local Voronoi cells near the source and the destination and this excessive path length contributes more to the total length when the network size is small.

The traditional UQS+LAR system selects shorter paths than our integrated scheme. This is because the LAR algorithm is able to perform path searching with the location information of the destination returned by querying the UQS system. It is important to note, however, that the destination's location must be obtained first, which adds to the routing time.

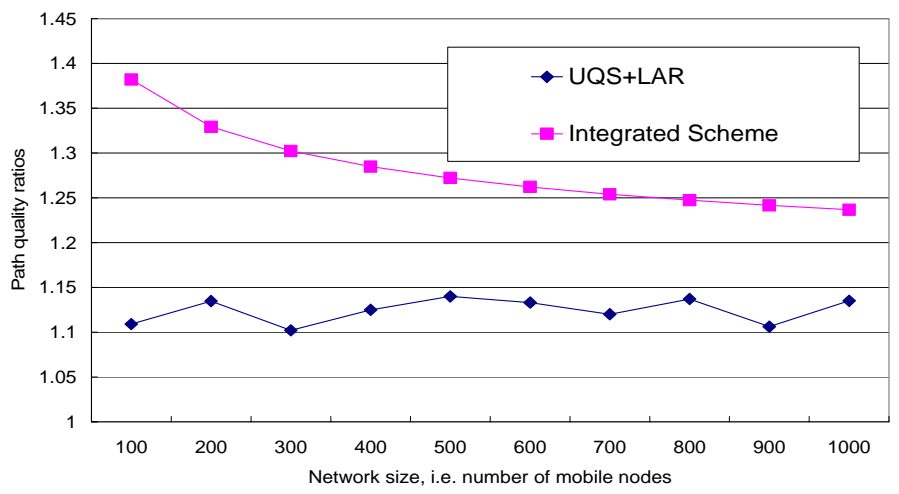

Fig. 4. Path ratio as a function of the number of nodes. Network area is fixed at $1000 \times 1000$.

The node density of the networks was kept constant while the area of the networks was varied in the second set of experiments. The results are listed in Table V.

\begin{tabular}{c|c|c|c|c|c} 
network size & shortest & UQS+LAR & $\begin{array}{c}\text { integrated } \\
\text { scheme }\end{array}$ & $\begin{array}{c}\text { UQS+LAR } \\
\text { phortest }\end{array}$ & $\frac{\text { Integrated }}{\text { shortest }}$ \\
\hline $1000 \times 1000\left(\mathrm{~m}^{2}\right)$ & 563.5 & 631.1 & 776.2 & 1.12 & 1.38 \\
\hline $2000 \times 2000\left(\mathrm{~m}^{2}\right)$ & 1108.3 & 1185.9 & 1252.8 & 1.07 & 1.13 \\
\hline $3000 \times 3000\left(\mathrm{~m}^{2}\right)$ & 1575.9 & 1654.7 & 1716.3 & 1.05 & 1.09
\end{tabular}

Table V. Path ratio as a function of network area. Node density is fixed at 100 nodes per $\mathrm{km}^{2}$.

While the paths are not as short as those in the UQS+LAR system, our scheme reduces the routing time significantly as shown in the following subsections. 
To determine the routing time ratio, we recorded the time that a packet took to go from a source to a destination in our integrated system $\left(T_{\text {integrate }}\right)$ and also in the UQS+LAR system ( $T_{\text {traditional }}$. Note that $T_{\text {traditional }}$ consists of two parts: $T_{\text {query }}$, the time for the source to query the destination's location, and $T_{\text {routing, }}$, the time to route the packet from source to destination. Therefore, the routing time ratio is given by:

$$
T_{\text {integrate }} /\left(T_{\text {query }}+T_{\text {routing }}\right) \text {. }
$$

As shown in Fig. 5, the time of our integrated location management and location-aided routing system is only $70-75 \%$ of the time for traditional systems. The main reason is that in our integrated system packets can be sent as soon as they are generated since the destination's location can be learned during routing. As indicated by the simulation results, by the time the traditional system is able to obtain the destination's location, the packet has almost finished half the journey to the destination. We note that for most users, the time taken for the packet to reach its destination is more important than whether the path taken is the shortest or not.

Note that the size of data transmission in ad hoc networks is usually small. The proposed integrated system works best in this scenario because the location query time in the traditional approach makes up a higher proportion of the total routing delay. However, if the number of packets sent between a source and the destination is large, the longer path taken by the integrated system may be a disadvantage. In this case, caching technique can be employed to create a hybrid system. The first several packets will be sent using the integrated system. Then the destination's location can be explicitly sent back to the source or piggybacked on a reply data packet. This location information is cached by the source for later packets to be directly routed using a location-aided routing algorithm, bypassing the location servers. The remaining packets therefore enjoy a shorter path to the destination. 


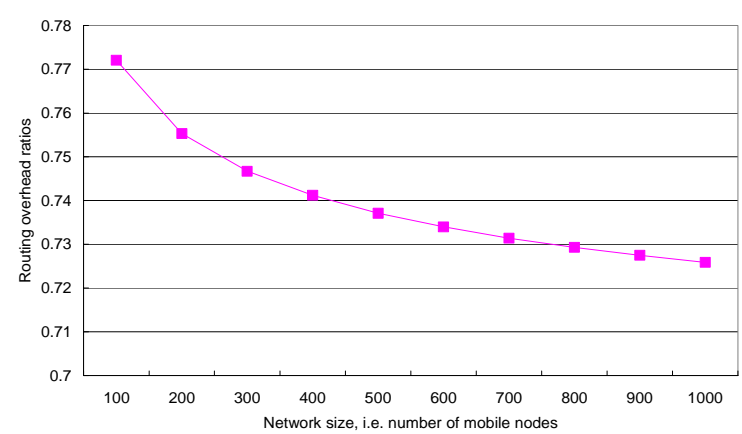

Fig. 5. Routing time ratios as a function of the number of nodes.

Network area was fixed at $1000 \times 1000$.

\subsubsection{Packet success ratio}

Fig. 6 gives the percentage of successful packet routing. We evaluated the system performance in two sets of experiments where the average node velocity was set at $2 \mathrm{~m} / \mathrm{s}$ and $10 \mathrm{~m} / \mathrm{s}$ respectively. The results show that the routing success rate decreases when the network size grows. This is due to the fact that more location servers are needed to relay the packets and the unavailability of any one will cause packet routing to fail. Another observation is that the routing success rate declines when node mobility is increased. This is caused by the higher frequency of node registration events. Furthermore, at high velocity, the topology of the network will vary more frequently and in some cases, isolated nodes may be formed.

Comparing to the UQS+LAR system, our integrated system shows better transmission success rate. This is because in the UQS+LAR system, the location information returned by UQS may become obsolete during routing by LAR, which increases the probability of routing failure. While in the integrated system, the location information of the destination node is learned during each packet routing procedure.

The performance difference between the two systems increases when the average node mobility is higher. The UQS+LAR system is more likely to return an outdate location information when nodes move faster, causing more packet transmissions to fail. 


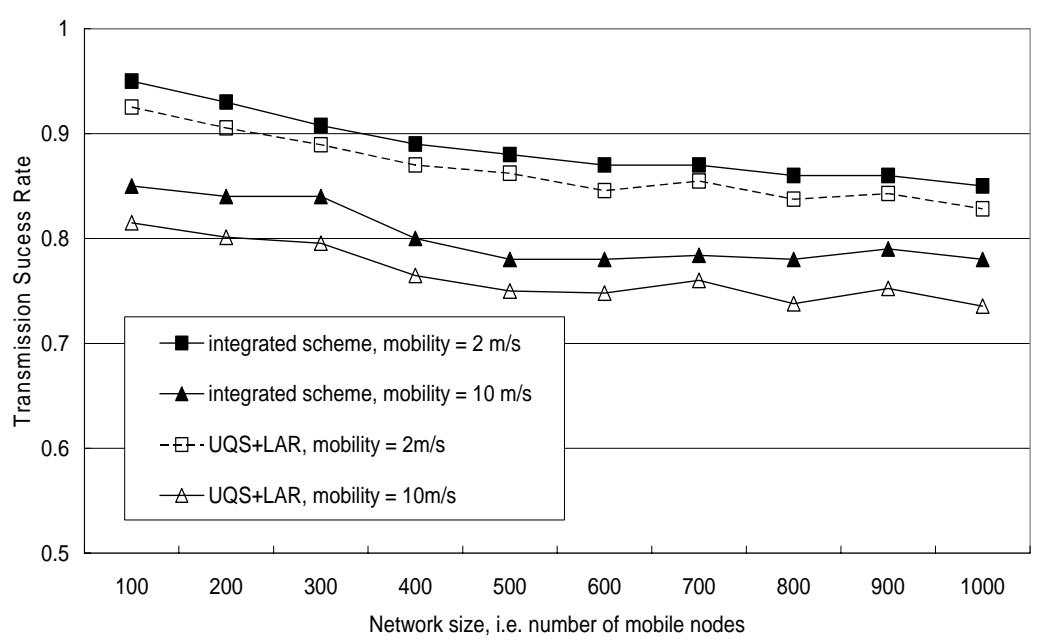

Fig. 6. Packet transmission success rate, with respect to network

size.

\subsubsection{Management overhead}

As mentioned in Section 4, management overhead is incurred by node registration, node location update and Bloom Filter update. Therefore, the frequency of these three events was measured and presented in Table VI as an indication of management overhead.

\begin{tabular}{c|c|c|c} 
network size & $\begin{array}{c}\text { node } \\
\text { registration }\end{array}$ & $\begin{array}{c}\text { node location } \\
\text { update }\end{array}$ & $\begin{array}{c}\text { Bloom filter } \\
\text { update }\end{array}$ \\
\hline $1000 \times 1000\left(\mathrm{~m}^{2}\right)$ & 0.312 & 10.57 & 3.15 \\
\hline $2000 \times 2000\left(\mathrm{~m}^{2}\right)$ & 0.320 & 10.63 & 6.41 \\
\hline $3000 \times 3000\left(\mathrm{~m}^{2}\right)$ & 0.323 & 10.81 & 9.72
\end{tabular}

Table VI. Average management overhead per node per second, in

fix density networks.

As shown in Table VI, the management overhead of the three networks was similar. This is mainly because they have similar node density and mobility velocity. It is seen that node location update occurs more frequently than the other two events. However it does not constitute a major part of the management overhead since node location update packets are only broadcasted within the local Voronoi zone. Furthermore, the size of the node location update message is small. The same argument holds for the node registration overhead.

To estimate the Bloom Filter update overhead among the location servers, the adhoc network 
with the largest size $\left(3000 \times 3000\left(\mathrm{~m}^{2}\right)\right)$ and the largest number of nodes $(900)$ was used. We estimated the bandwidth utilization for a $2 \mathrm{Mbps}$ link from a location server's point of view. The Bloom Filter update has to be distributed to all the neighboring location servers. If we use a 450-byte Bloom Filter to represent the 900 nodes (i.e., $b / N=4$ ), the estimated bandwidth utilization was less than $7 \%$.

\section{Conclusion}

We have presented an integrated location management and location-aided routing system in this paper. In our scheme, location information of the mobile nodes is kept in an overlay network of location servers. Routing is performed in the two-level hierarchy. The destination's location is learned during routing in the overlay network and used to guide the packet toward the destination.

Both theoretical analyses and simulation results have shown that the proposed system reduces the time to route short packets to the destination compared to traditional location management systems. The final path adopted by the new system approximates the shortest path well. The location update messages are sent to nearby location servers only. Furthermore, message exchanges among location servers are reduced to the minimum by the use of Bloom Filters. Therefore both network bandwidth and storage requirement of the proposed system are low.

\section{References}

[1] F. Aurenhammer, Voronoi Diagrams - A Survey of a Fundamental Geometric Data Structure, ACM Computing Surveys, Vol 23, p 345- 405 (1991).

[2] F.Baccelli, K.Tchoumatchenko, S.Zuyev. Markov Paths on the Poisson-Delaunay Graph. INRIA Research Report No. 3420. Mai.1998.

[3] S. Basagni, I. Chlamtac, V. R. Syrotiuk, and B. A. Woodward. A distance routing effect algorithm for mobility (DREAM). In Proceedings of the Fourth Annual ACM/IEEE International Conference on Mobile Computing and Networking, MobiCom'98, pages 76--84, Dallas, TX, October 25--30,1998.

[4] A. Broder, M. Mitzenmacher, Network Applications of Bloom Filters: A Survey, http://citeseer.ist.psu.edu/543187.html. 
[5] S. Fortune. Voronoi diagrams and Delaunay triangulations. In Du D.-Z. and Hwang F., editors, Computing in Euclidean Geometry, volume 4 of Lecture notes series on Computing, pages 225--265. World Scientific, Singapore, 2nd edition, 1995.

[6] G. Giordano, M. Hamdi, Mobility Management: The Virtual Home Region, Technical Report No. SSC/1999/037, EPFL, October 1999.

[7] Glomosim simulator homepage, http://pcl.cs.ucla.edu/projects/glomosim/.

[8] M. Grossglauser and M. Vetterli. Locating Nodes with EASE: Mobility Diffusion of Last Encounters in Ad Hoc Networks. In Proc. IEEE INFOCOM '2003.

[9] Z. J. Haas and B. Liang, Ad-Hoc Mobility Management with Uniform Quorum Systems, IEEE/ACM Transactions on Networks, 7(2), pp. 228-240, 1999.

[10] X. Hong, M. Gerla, L. Ma, Multiple Landmark Routing for Large Groups in Ad Hoc Networks, in Proceedings of MilCom 2002.

[11] T. Hou, V. Li, Transmission Range Control in Multihop Packet Radio Networks, IEEE Transaction on Communications, 34(1): 38-44, Jan. 1986.

[12] IETF MANET charter, http://www.ietf.org/html.charters/manet-charter.html.

[13]B. Karp, H. Kung, Greedy Perimeter Stateless Routing (GPSR) for Wireless Networks, In Proc. Sixth Annual ACM/IEEE International Conference on Mobile Computing and Networking (MobiCom), pages 243-254, 2000.

[14] L. Kleinrock, J. Silvester, Optimum Transmission Radii for Packet Radio Networks or Why Six is a Magic Number, in Proceedings of IEEE National Telecommunications Conference, 1978, pp. 431-435.

[15] Y. Ko, N. Vaidya, Location-Aided Routing (LAR) in Mobile Ad Hoc Networks, ACM/Baltzer Wireless Networks (WINET) journal, 6(4) : 307-321, 2000.

[16] J. Li, J. Jannotti, D. Decouto, D. Karger, and R. Morris, A scalable location service for geographic ad-hoc routing. In Proceedings of the Sixth Annual ACM/IEEE International Conference on Mobile Computing and Networking (MobiCom 2000) (Boston, MA, USA, Aug. 2000).

[17]W. Li, G. Calinescu, P. Wan, Y. Wang, Localized Delaunay Triangulation with Applications in Ad-hoc Wireless Networks, IEEE Transaction on Parallel and Distributed Systems, October 2003 (Vol. 14, No. 10), pp. 1035-1047.

[18] J. Liu, X. Zhang, B. Li, Q. Zhang, and W. Zhu, Distributed Distance Estimation for Large-Scale Networks, Elsevier Computer Networks, Vol. 41, No. 2, pp. 177-193, February 2003. 
[19] M. Mauve, J. Widmer, H. Hartenstein, A Survey on Position-based Routing in Mobile Ad-Hoc Networks, IEEE Network Magazine, 15(6): 30-39, Nov. 2001.

[20] M. Mitzenmacher. Compressed bloom filters. In Twentieth ACM Symposium on Principles of Distributed Computing (PODC 2001).

[21] G. Pei, M. Geda, and X. Hong, Lanmar: Landmark routing for large scale wireless ad hoc networks with group mobility, In ACM MobiHoc, August 2000.

[22] E. Royer, C. Toh, A Review of Current Routing Protocols for Ad-Hoc Mobile Wireless Networks, IEEE Personal Communications, Apr. 1999, pp. 46-55.

[23] L. Stojmenovic, Home Agent Based Location Update and Destination Search Schemes in Ad-hoc Wireless Networks, Technical report TR-99-10, Computer Science, SITE, University of Ottawa, Sept. 1999.

[24]H. Takagi, L. Kleinrock, Optimal Transmission Ranges for Randomly Distributed Packet Radio Terminals, IEEE Transaction on Communications, 32(3): 246-257, Mar. 1984.

[25] P. F. Tsuchiya, The Landmark Hierarchy: A New Hierarchy for Routing in Very Large Networks, ACM SIGCOMM 1988.

[26] B. Y. Zhao, Y. Duan, L. Huang, A. Joseph, and J. Kubiatowicz, Brocade: Landmark Routing on Overlay Networks. In Proceedings of 1st International Workshop on Peer-to-Peer Systems (IPTPS) (March 2002). 